\title{
Development of drug-loaded magneto-sensitive liposomes investigated by fluorescence techniques
}

Beatriz D. Cardoso, Daniela S. M. Pereira, A. Rita O. Rodrigues, Paulo J. G. Coutinho and Elisabete M. S. Castanheira

Centre of Physics (CFUM), University of Minho, Campus de Gualtar, 4710-057 Braga, Portugal

Magneto-sensitive liposomes can be obtained by encapsulation of magnetic nanoparticles into liposomes or by the coverage of magnetic nanoparticles with a lipid bilayer. The so-called magnetoliposomes make possible to explore the synergistic effect between chemotherapy and magnetic hyperthermia in cancer therapeutics. Both aqueous magnetoliposomes (magnetic nanoparticles entrapped in liposomes) and solid magnetoliposomes (clusters of nanoparticles covered by a lipid bilayer), containing biocompatible magnetic nanoparticles, have been developed [1-3], exhibiting a superparamagnetic behavior and diameters below $150 \mathrm{~nm}$.

These nanosystems were successfully tested as nanocarriers for fluorescent potential antitumor drugs. Drug-loaded magnetoliposomes have shown the ability to interact by fusion with models of biomembranes [1-3] and to release the antitumor drugs in in vitro assays using human tumour cell lines [2]. Fluorescence-based methodologies, including Förster Resonance Energy Transfer (FRET), emission quenching and fluorescence anisotropy, have been used as valuable tools for this investigation.

Acknowledgements: FCT, FEDER, PORTUGAL2020 and COMPETE2020 for funding under Project PTDC/QUI-QFI/28020/2017 (POCl-01-0145-FEDER-028020) and Strategic funding UID/FIS/04650/2013. B.D.C. acknowledges FCT for a PhD grant (SFRH/BD/141936/2018).

[1] A.R.O. Rodrigues et al., Colloid Surfaces B-Biointerfaces 2017, 158, 460.

[2] A.R.O. Rodrigues et al., RSC Advances 2017, 7, 15352.

[3] B.D. Cardoso et al. Royal Society Open Science, 2018, 5, 181017. 